\title{
A Reduction of Lattice Tiling by Translates of a Cubical Cluster
}

\author{
Sándor Szabó \\ Department of Civil Engineering Mathematics, Technical University of Budapest, \\ H-1111 Budapest, Stoczek u.2., Hungary
}

\begin{abstract}
A cluster is the union of a finite number of cubes from the standard partition of $n$-dimensional Euclidean space into unit cubes. If there is lattice tiling by translates of a cluster, then must there be a lattice tiling by translates of the cluster in which the translation vectors have only integer coordinates? In this article we prove that if the interior of the cluster is connected and the dimension is at most three, then the answer is affirmative.
\end{abstract}

Let $e_{1}, \ldots, e_{n}$ be the coordinate unit vectors in $n$-dimensional Euclidean space $R^{n}$ and let 0 be the origin of this coordinate system. Take a finite set of vectors $T$ such that $0 \in T$ and each element of $T$ has only integer coordinates. The union of translates by the vectors of $T$ of a unit cube whose edges are parallel to $e_{1}, \ldots, e_{n}$ we will denote by $\mathscr{T}_{0}$. For the set of translates of $\mathscr{T}_{0}$ by the elements of the vector set $L$ we will use the notation $\left(\mathscr{T}_{0}, L\right)$. If the elements of $\left(\mathscr{T}_{0}, L\right)$ cover $R^{n}$ and have no common interiors, then we will speak of a tiling. We will also speak of lattice and integer (rational) tiling depending on whether $L$ is a lattice or each element of $L$ has only integer (rational) coordinates, respectively.

When the cluster $\mathscr{T}_{0}$ is a so-called $(k, n)$ cross or semicross, that is, when $T=\left\{i e_{j}:|i| \leq k, 1 \leq j \leq n\right\}$ or $T=\left\{i e_{j}: 0 \leq i \leq k, 1 \leq j \leq n\right\}$, the existence of the corresponding tiling $\left(\mathscr{T}_{0}, L\right)$ has been investigated in a series of papers (see [3]). This is the motivation of the investigation in which there is no restriction on $\mathscr{F}_{0}$. The following known facts about the existence of $\left(\mathscr{T}_{0}, L\right)$ will be needed.

If there is a lattice tiling $\left(\mathscr{T}_{0}, L\right)$, then there exists a rational lattice tiling $\left(\mathscr{T}_{0}, L^{\prime}\right)$ (see [4]).

If there is a tiling $\left(\mathscr{T}_{0}, L\right)$ then there exists an integer tiling $\left(\mathscr{T}_{0}, L^{\prime}\right)$ (see [1]). The next statement would be a common generalization. If there is a lattice tiling $\left(\mathscr{T}_{0}, L\right)$ then there is an integer lattice tiling $\left(\mathscr{T}_{0}, L^{\prime}\right)$. But this is not true for $n \geq 2$ as the following example shows. Let $T$ be $\left\{0,2 e_{1}\right\}$; the corresponding $\mathscr{T}_{0}$ consists of two disconnected $\boldsymbol{n}$-dimensional unit cubes. If $L$ is a lattice spanned 
by the vectors $4 e_{1}, e_{1}+\frac{1}{2} e_{2}, e_{2}, e_{3}, \ldots, e_{n}$, then $\left(\mathscr{T}_{0}, L\right)$ is a lattice tiling and if $\left(\mathscr{T}_{0}, L^{\prime}\right)$ had been an integer lattice tiling, then $e_{1}$ would have been an element of $L^{\prime}$ and this is a contradiction since $2 e_{1}$ cannot be in $L^{\prime}$.

The previous example is due to Hickerson and in connection with this in [2] he asked whether this phenomenon could occur for connected sets, such as crosses and semicrosses. The next theorem provides a partial answer.

Theorem. If the interior of $\mathscr{T}_{0}$ is a connected set and there is an n-dimensional lattice tiling $\left(\mathscr{T}_{0}, L\right)$ where $n \leq 3$, then there is an integer lattice tiling $\left(\mathscr{T}_{0}, L^{\prime}\right)$.

Proof. We define an equivalence relation on $\left(\mathscr{T}_{0}, L\right)$. If $\mathscr{T}_{A}, \mathscr{T}_{B} \in\left(\mathscr{T}_{0}, L\right)$ and the $i$ th coordinate of $\overrightarrow{A B}$ is an integer, then $\mathscr{T}_{A}$ and $\mathscr{T}_{B}$ are in the same equivalence class. A property of these equivalence classes will play an important role in this proof. Namely, each equivalence class can be translated freely in $\left(\mathscr{T}_{0}, L\right)$ in the direction of $e_{i}$ as a rigid body. In other words, the union of the elements of an equivalence class is an infinite prism in the direction of $e_{i}$. The proof can be found in [4], Theorem 5 .

If a subset $\left(\mathscr{T}_{0}, K\right)$ of $\left(\mathscr{T}_{0}, L\right)$ can be translated freely in $\left(\mathscr{T}_{0}, L\right)$ in the direction of $e_{i}$ as a rigid body, then we will call $\left(\mathscr{T}_{0}, K\right)$ an $i$-prism. If each proper part of an i-prism is not an $i$-prism, then we will call it an irreducible i-prism.

Note that if $\left(\mathscr{T}_{0}, K\right)$ is an irreducible $i$-prism, then the $i$ th coordinates of elements of $K$ differ by integers.

Obviously, according to the latticity of $\left(\mathscr{T}_{0}, L\right)$ the irreducible $i$-prism are translates of each other so we will investigate that copy which contains the point 0 .

(i) If $\left(\mathscr{T}_{0}, K\right)$ is an irreducible i-prism and $0 \in K$, then $K$ is a lattice.

Indeed, let $a$ and $b$ be elements of $K$. To prove that $a-b \in K$, note that the vector $a-b$ translates $\left(\mathscr{T}_{0}, L\right)$ onto itself and prisms go into prisms. Since the intersection of nondisjoint prisms is a prism as well, the irreducible prism are either disjoint or identical. Obviously, $\mathscr{T}_{A} \in\left(\mathscr{T}_{0}, K\right) \cap\left(\mathscr{T}_{0}, K+a-b\right)$ so $\left(\mathscr{T}_{0}, K\right)=\left(\mathscr{T}_{0}, K+a-b\right)$, that is, $a-b \in K$.

If $\left(\mathscr{T}_{0}, K\right)$ is an irreducible $i$-prism and $\mathscr{T}_{A} \in\left(\mathscr{T}_{0}, K\right)$, then there has to be an element of $\left(\mathscr{T}_{0}, K\right)$ which blocks $\mathscr{T}_{A}$ against a translation in the direction of $e_{i}$ since otherwise $\left(\mathscr{T}_{0}, K\right) \backslash \mathscr{T}_{A}$ would have been an $i$-prism. Continuing in this way and using the fact that the interior of $\mathscr{T}_{0}$ is connected we obtain:

(ii) If $\left(\mathscr{T}_{0}, K\right)$ is an irreducible $i$-prism and $\mathscr{T}_{A}, \mathscr{T}_{B} \in\left(\mathscr{T}_{0}, K\right)$, then there exists a polygon from $A$ to $B$ lying in the strict interior of the union of elements of $\left(\mathscr{T}_{0}, K\right)$.

In fact, as the referee has pointed out, we obtained new descriptions of irreducible $i$-prisms. For $\mathscr{T}_{A}, \mathscr{T}_{B} \in\left(\mathscr{T}_{0}, L\right), \mathscr{T}_{A}$ and $\mathscr{T}_{B}$ are in the same irreducible $i$-prism if and only if there exist $A=A_{0}, A_{1}, \ldots, A_{n}=B$ such that $\mathscr{T}_{A_{l}}$ and $\mathscr{T}_{A_{i+1}}$ share part of a face perpendicular to $e_{i}, 0 \leq i \leq n-1$.

If $L$ is a lattice and $L^{\prime}$ is a sublattice of $L$ and there exists another sublattice $L^{\prime \prime}$ such that $L=L^{\prime}+L^{\prime \prime}$ and $L^{\prime} \cap L^{\prime \prime}=\{0\}$, then we will say $L^{\prime}$ is a direct summand of $L$.

If $\mathscr{T}_{0}$ has the property that for each lattice tiling $\left(\mathscr{T}_{0}, L\right)$ for each irreducible prism $\left(\mathscr{T}_{0}, K\right)$ with $0 \in K, K$ is a direct summand of $L$, then we can complete the proof in the following way. 
Let $\left(\mathscr{T}_{0}, K_{1}\right)$ be an irreducible 1-prism with $0 \in K_{1}$ and let $K_{1}^{\prime}$ be the direct summand pair of $K_{1} . K_{1}^{\prime}$ is spanned by vectors $k_{i}^{\prime}=a_{i 1} e_{1}+\cdots+a_{i n} e_{n}, 1 \leq i \leq t$. Replace $k_{1}^{\prime}, \ldots, k_{t}^{\prime}$ by $k_{1}^{*}=k_{1}^{\prime}-a_{11} e_{1}, \ldots, k_{t}^{*}=k_{t}^{\prime}-a_{t 1} e_{1}$, respectively. We get a new lattice $L_{1}=K_{1}+K_{1}^{*}$. The system $\left(\mathscr{T}_{0}, L_{1}\right)$ is a tiling. Indeed, since $L_{1}=$ $K_{1}+K_{1}^{*}, L=K_{1}+K_{1}^{\prime},\left(\mathscr{T}_{0}, L\right)$ and $\left(\mathscr{T}_{0}, L_{1}\right)$ consists of translates of $\left(\mathscr{T}_{0}, K_{1}\right)$ and since $K_{1}+x_{1} k_{1}^{*}+\cdots+x_{t} k_{t}^{*}=K_{1}+x_{1} k_{1}^{\prime}+\cdots+x_{t} k_{t}^{\prime}-\left(x_{1} a_{11}+\cdots+x_{t} a_{11}\right) e_{1}$, the 1-prisms are translated only in the direction of $e_{1}$.

Note that the first coordinates of elements of $L_{1}$ are integers. Repeat this procedure for the $2 \mathrm{nd}, 3 \mathrm{rd}, \ldots, n$th coordinates. Finally, we get an integer lattice tiling by translates of $\mathscr{T}_{0}$.

Take an $n$-dimensional lattice tiling $\left(\mathscr{T}_{0}, L\right)$ where $n \leq 3$ and assume that the interior of $\mathscr{T}_{0}$ is connected. We shall prove that in this case $\left(\mathscr{T}_{0}, L\right)$ has the property which was assumed in the previous consideration. For the sake of simplicity we will restrict the proof to the three-dimensional case and 1-prisms. The next statement represents the most important step toward the complete proof.

(iii) If $n=3$ and $L^{\prime}$ is a one-dimensional sublattice of $L$ then either each element of the system $\left(\mathscr{T}_{0}, L^{\prime}\right)$ belongs to the same irreducible 1-prism or no two belong to the same irreducible 1-prism.

To prove (iii), assume the contrary: $\mathscr{T}_{0}, \mathscr{T}_{A}, \mathscr{T}_{B} \in\left(\mathscr{T}_{0}, L^{\prime}\right)$ and $\mathscr{T}_{0}, \mathscr{T}_{A}$ are in the same irreducible 1-prism and $\mathscr{T}_{B}$ belongs to another. If $\overrightarrow{O A}$ is parallel to $e_{1}$ then we are done. So we may assume that $\overrightarrow{O A}$ is not parallel to $e_{1}$. Let $L^{\prime \prime}$ be the lattice spanned by the vector $\overrightarrow{O A}$. According to (i) the elements of the system $\left(\mathscr{T}_{0}, L^{\prime \prime}\right)$ belong to the same irreducible 1-prism. According to (ii) there is a polygon from $O$ to $A$. Consequently, translating it by $\overrightarrow{j O A}, j \in Z$ we get an infinitely periodic polygon within the strict interior of an irreducible 1-prism. Since the system $\left(\mathscr{T}_{B}, L^{\prime \prime}\right)$ is a translate of $\left(\mathscr{T}_{0}, L^{\prime \prime}\right)$, the elements of $\left(\mathscr{T}_{B}, L^{\prime \prime}\right)$ belong to the same irreducible 1-prism which is different from the previous one and the translate of the polygon is in the strict interior of the new 1-prism. Project the polygons into a plane perpendicular to $e_{1}$. We get two infinitely periodic polygons which are translates of each other into the direction of their common period. Hence there exists a smallest common strip which contains the polygons. The polygons intersect each other since both of them connect and separate the borders of the strip. The points of a line which is parallel to $e_{1}$ and pass through the points of our polygons have to be covered by the same irreducible 1-prism. When we take a line through the common point of the projection of the polygons which is parallel to $e_{1}$, we get two irreducible 1-prisms such that the strict interiors of their unions are not disjoint. This is a contradiction.

Assume that $\left(\mathscr{T}_{0}, L\right)$ is a three-dimensional lattice tiling and the interior of $\mathscr{T}_{0}$ is connected and $L$ is spanned by $l_{1}, l_{2}$, and $l_{3}$. By the last step apply (iii) to the lattices $L^{\prime}, L^{\prime \prime}$, and $L^{\prime \prime \prime}$ spanned by $l_{1}, l_{2}$, and $l_{3}$, respectively; so we conclude that for each irreducible 1-prism $\left(\mathscr{T}_{0}, K\right), K$ is a direct summand of $L$ which completes the proof.

\section{Acknowledgment}

The author would like to thank the referee for his valuable suggestions. 


\section{References}

1. H. Everett and D. Hickerson, Packing and covering by translates of certain nonconvex bodies, Proc. Amer. Math. Soc. 75 (1979), 87-91.

2. D. Hickerson, Splittings of finite groups, Pacific J. Math. 107 (1983), 141-171.

3. S. K. Stein, Tiling, packing and covering by clusters, Rocky Mountain J. Math. 16 (1986), 277-321.

4. S. Szabó, On mosaics consisting of multidimensional crosses, Acta Math. Acad. Sci. Hungar. 39 (1981), 191-203.

Received July 11, 1985, and in revised form October 7, 1985. 